necessity was later disputed by Naidu (J. geophys. Res., 76, 3426; 1971) but reaffirmed by Horai and Nur (J. geophys. Res., 76, 3428; 1971). A further conflict then came to light when it became clear that although Horai and Nur had found that the positive $q-k$ correlation applied to the Indian peninsular shield, Gupta and Rao (Bull. natn. Geophys. Res. Inst., 8, 87; 1970) had already found no such correlation in the Indian Precambrian crystalline regions.

Continuing along similar lines, and to make matters even more confused, Negi and his coworkers have found that for all Indian sedimentary basins $q$ correlates with $k$ negatively. On the other hand, a positive correlation between heat flow and geothermal gradient $(G)$ agrees with that found by Gupta and Rao for Indian Precambrian crystalline areas. Finally, heat flow is found to correlate negatively with crustal thickness.

What does it all mean? Negi et al. conclude that for sedimentary basins, at least, the negative correlation between $q$ and $k$ implies very little heat generation in the crust; in other words, most of the observed heat comes from below. The observed correlations between $q$, $k, G$ and crustal thickness are often presumed to be due to variations in crustal thickness which produce a variable shielding effect on subcrustal heat. These are rather mundane conclusions to emerge from so much work; and it is difficult to judge their importance. Clearly, however, the parameters involved are very basic to the whole question of variations in heat flow; and so it would be helpful if someone would put all the data together and, if possible, explain just what is to be made of them.

\section{Semiconductors in the human body?}

from our

Solid State Physics Correspondent

IT was realised about 20 years ago that the production of energy from oxygen in the cell mitochondrion was possibly the result of direct electronic transport through haemoproteins rather than the interaction of mobile ions through an aqueous medium (see, for example, Szent-Györgyi, Discuss. Faraday Soc., 27, 111; 1959; Carden and Eley, ibid., 115). It was thus necessary to think about a sort of wet solid-state physics which could embrace reasonably high rates of transport of free electrons or holes through biological solids, especially through certain haemoproteins involved in respiration. Szent-Györgyi proposed a theory that a suitable conduction band could be produced by orbital overlaps in amino acid chains and one could predict from this theory that proteins might achieve conductivities in the semiconductor range. The theory was conceptually very attractive but was not widely taken up, largely because it was not at the time susceptible of proof, nor had any investigation ever revealed a way of making analogous but simpler organic materials conduct to the level required.

Now, at least, one biological material has been shown to have a strikingly large conductivity when correctly excited. McGinness, Corry and Proctor, of the University of Texas Cancer Center, Houston, report in Science $(183,853 ;$ 1974) that melanins can be made to 'switch' from a poorly conducting to a highly conducting state at fairly low electric fields (say from $10^{5} \Omega$ $\mathrm{cm}$ to $10^{2} \Omega \mathrm{cm}$ at a field of $3 \times 10^{2} \mathrm{~V}$ $\mathrm{cm}^{-1}$ ). This remarkable phenomenon occurs both in melanin made synthetically from tyrosine and in that extracted from a human melanoma. The large conduction is not destructive in any way and is reversible; according to some tests, conduction seems to be electronic rather than ionic. Also tests of a few other likely biological materials in the same form (a compressed solid pellet inside a quartz tube, mildly hydrated and of length ranging from 0.1 to $10 \mathrm{~mm}$ ) suggest that the effect is confined to the melanins and the authors note a similarity in the $I-V$ characteristics of the sample to those of some amorphous inorganic semiconductors which undergo 'threshold switching'. But apart from the major difference in the electric field at which the threshold effect occurs (of the order of $10^{2} \mathrm{~V} \mathrm{~cm} \mathrm{~cm}^{-1}$ for melanin and $10^{5}$

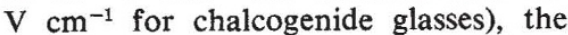
current theory of the inorganic switching phenomenon rests on filamentary conduction, leading to a controlled degree of segregation of the constituents of the glass (for example, segregation of pure tellarium from $\mathrm{Ge}-\mathrm{Te}$ alloys) and possibly strong injection at the electrodes under the high local fields (Bosell and Thomas, Phil. Mag., 27, 665-81; 1973).

Neither of these effects seems even likely in the system described, especially since the switching becomes unstable at thicknesses of chalcogenide greater than a few micrometres. Thus, the suggestion of McGuiness et al. that melanin in the human body can be a cause rather than a by-product of disease and that its mode of action can be related to this 'electronic device' action is probably premature, especially considering the preliminary nature of the experiments. A revivial of discussion on in vivo electronic effects in some biological materials associated with oxidation-reduction is, however, welcome if only because science has perhaps moved far enough since the 1950s that it can now devise adequate tests for the basic theories of transport in wet solids. Also a new approach to the treatment of melanotic diseases may well be stimulated by this particular revival of an intellectually stimulating discussion.

\section{How to wrap up and get your female}

\section{from our}

Animal Behaviour Correspondent

MichaEL and Barbara Robinson spent a year in New Guinea observing the giant wood spider, Nephila maculata (Fabricius), Their report (Smithson. Contrib. Zool. No. 149; 1973) contains some remarkable facts of spider life, including a description of the strange method of courtship. As can be seen from the photograph, the male is much smaller than the female $(4-6 \mathrm{~mm}$ body length as opposed to $40-50 \mathrm{~mm}$ ) and before actual copulation, he goes through an elaborate 'lashing' of the female, weaving silk onto her 'body. The male may spend the greater part of two days standing on the female and during this time periodically indulges in intense bursts of activity, laying down silk threads between the female's legs and body. The female rarely gives any response at all to this silk deposition, after which the male copulates.

It would seem logical to suppose that the functional significance of binding up the female in this way would be that it in some way restrains her and prevents her from attacking the male. But although a great deal of silk is laid down around the leg bases, this does not seem to hamper the female very much, for she can rush off on predatory excursions even after the silk

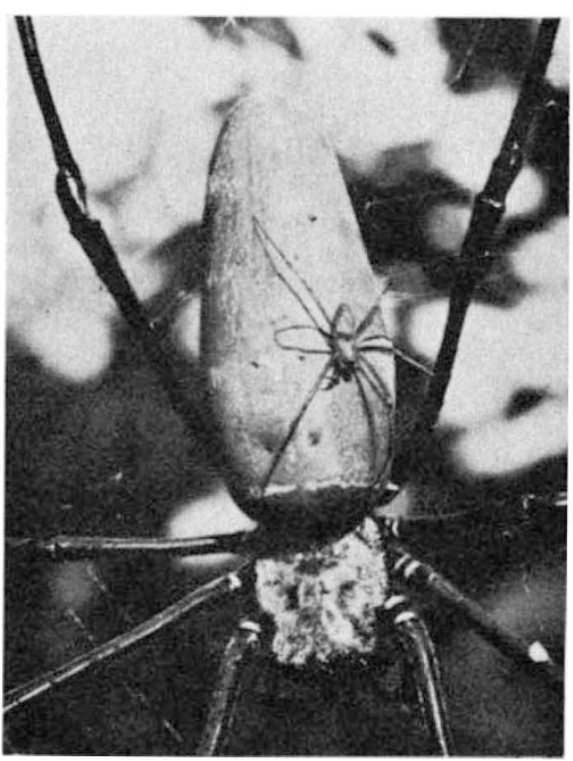

Male on dorsal surface of the abdomen of a female Nephila maculata. Silk lines are visible on the thorax of the female leading to the leg bases and abdomen. 